\author{
Military Technical College \\ Kobry Elkobbah, \\ Cairo, Egypt
}

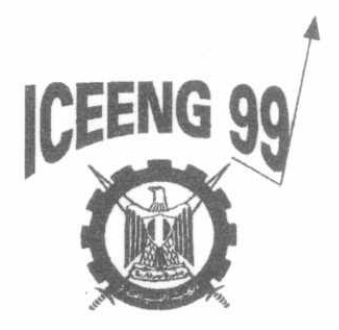
$2^{\text {nd }}$ International Conference on Electrical Engineering ICEENG 99

\title{
DESIGN,IMPLEMENTATION AND VERIFICATION OF C-BAND GAAS MESFET OSCILLATOR
}

\author{
ABDALLA* M.I., MOSELHY ${ }^{* *}$ A.M., ABDEL-NAZEER ${ }^{* * *}$ A., MAGHAWRY**** M.
}

\begin{abstract}
:
In this paper, the Gain Saturation Approximation method is used for the design of a 4.5 GHz GaAs MESFET Oscillator. This method is based on S-Parameter simulation of the initial oscillator topology including the transistor model. A computer-aideddesign was employed for optimizing and simulating the designed circuit to obtain the conditions of oscillation.

To verify this work, the optimized circuit is fabricated, by using microstrip technology, and measured. The optimized circuit is also simulated using MDS program. Good agreement between simulation and measurement is obtained.
\end{abstract}

\section{KEY WORDS:}

Microwave, Microstrip, Oscillator, CAD, and C-band

\footnotetext{
* Associate Professor, Comm. Department, Zagazig University, Zagazig, Egypt.

** Ph. D., Comm. Department, Zagazig University, Zagazig, Egypt.

*** Ph. D., Armed Forces, Egypt.

**** Graduate student, Armed Forces, Egypt.
} 


\section{INTRODUCTION:}

The generation of microwave signals has generally been performed using vacuum tubes and solid-state devices. Tubes such as klystrons and traveling-wave tubes are excessively used for high power applications, whereas solid-state devices are suitable for low noise, low and medium power levels. Microstrip solid-state oscillators are particularly useful for aerospace and military applications where weight and size impose severe limitations on the design of the system.

The design approach presented in this paper is based on the advanced techniques available in modern CAD programs.

\section{VERIFYING THE TRANSISTOR MODEL:}

For small signal linear behavior, the components in the schematic of Fig.1 are derived as constant values, which represent behavior at the quiescent operating point of the device $[1,2]$. We make a verification to our transistor MESFET(P351105-1) by using the available MDS.

The S-Parameter verification model for this transistor is shown in Fig.2. First, the transistor model is implemented using the microwave design system. Second, this model is simulated to obtain its S-Parameters. The simulation output of (P35-1105-1) MESFET transistor is shown in Fig.3.

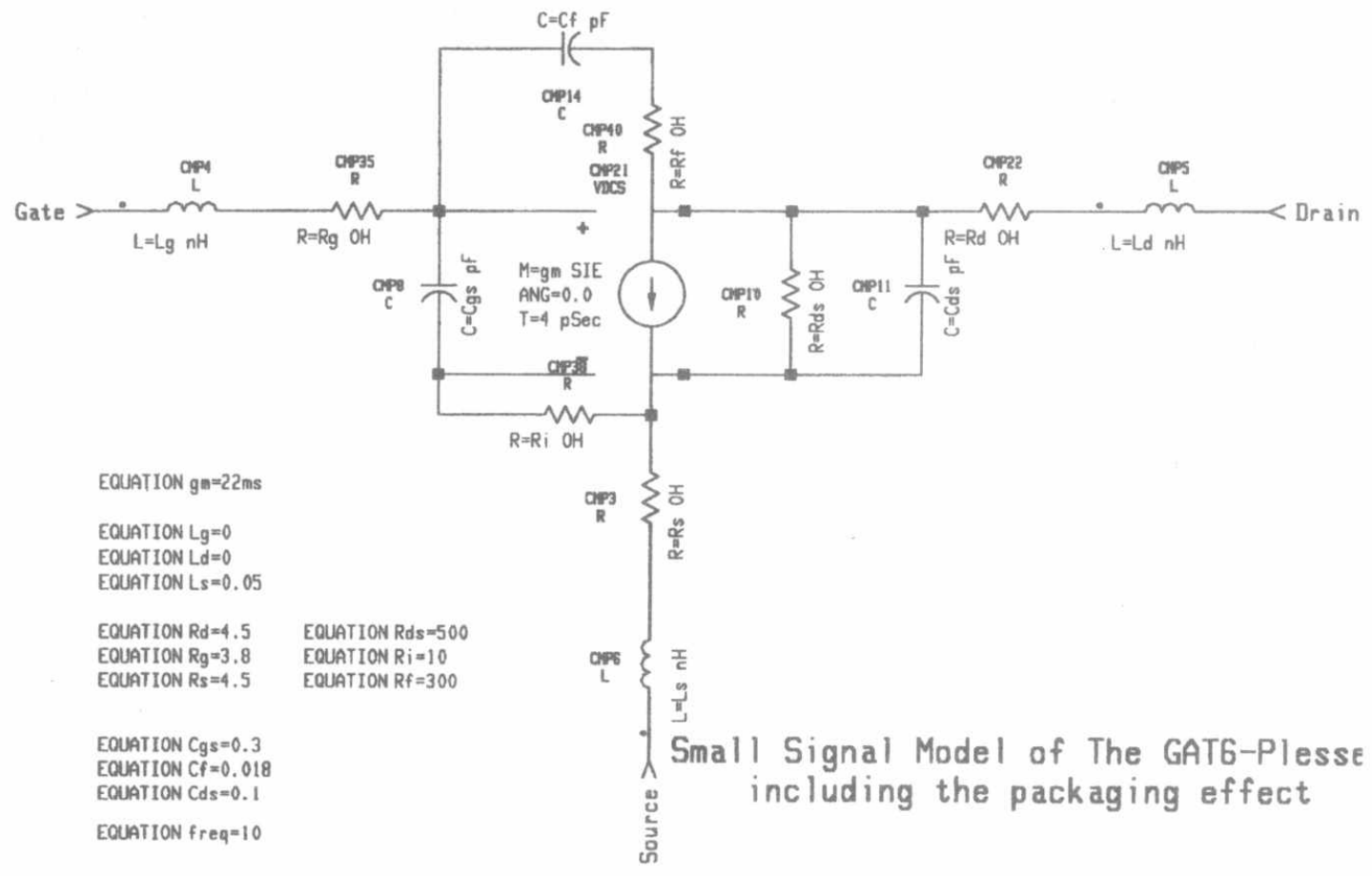

Fig.1 Small signal model of the GAT6- Plessey transistor, including packaging effect. 


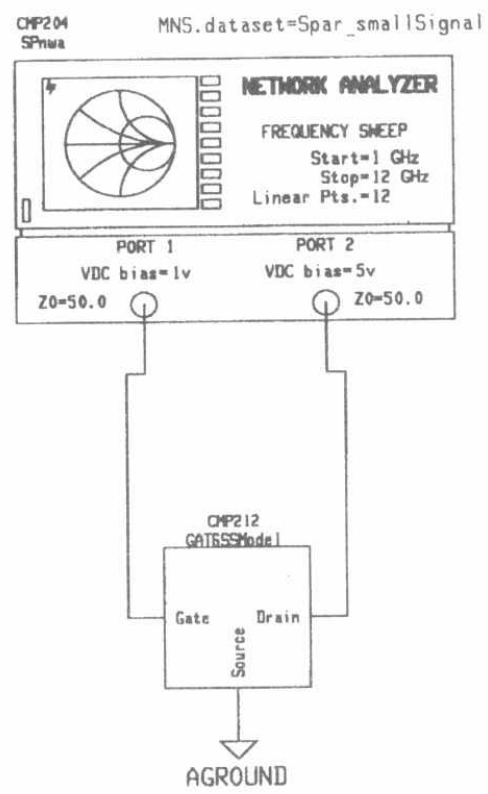

Fig.2 S-parameter Simulation of the implemented GAT6 transistor.

\section{THE GAIN SATURATION APPROXIMATION AND OSCILLATOR CIRCUIT DESIGN:}

We know that the device is characterized initially with Small-signal S-Parameters. Johnson [3] has suggested a simple approach for modeling the large-signal power. The primary change in the device can be characterized by a reduction in magnitude of $\mathrm{S}_{21}$, the other S-Parameters are not change. The new value of $\mathrm{S}_{21}$ is corresponding to maximum oscillator output power and we use the new value to design our oscillator.

$$
P_{\text {out }} \cong P_{S A T}\left\{1-\exp \left(\frac{-G o P_{i n}}{P_{\text {sat }}}\right)\right\}
$$

Where:

$G_{O}$ Small signal gain

$P_{\text {sat }}$ Saturated output power as an amplifier

$$
G_{M E}=\frac{\left|\frac{S_{21}}{S_{12}}\right|^{2}-1}{2\left\{K\left|\frac{S_{21}}{S_{12}}\right|-1\right\}}
$$

Where:

$G_{M E} \quad$.......... Maximum efficient gain

$K \quad$......... The rollett stability factor 


$$
\text { 萿 }
$$
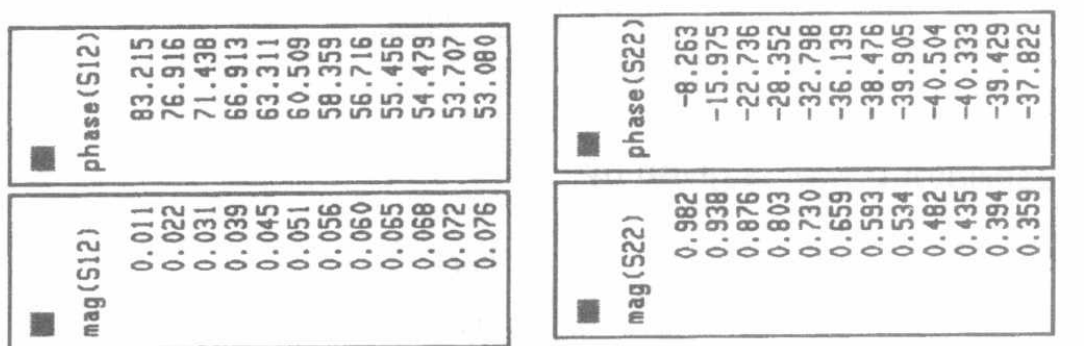

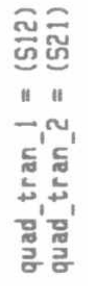
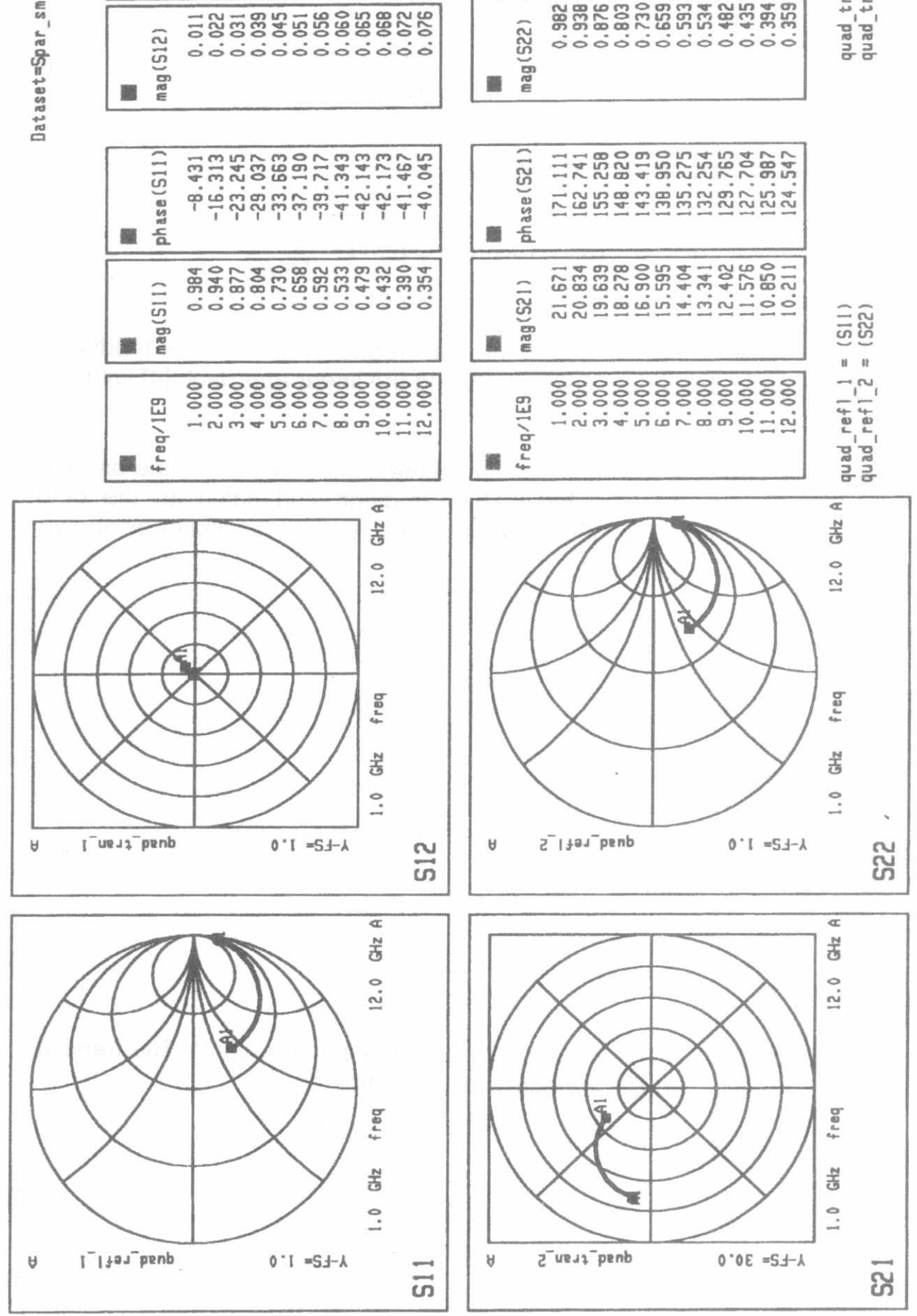

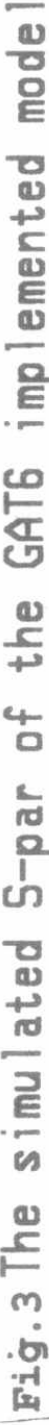




$$
K=\frac{1+\left|S_{11} S_{22}-S_{21} S_{12}\right|^{2}-\left|S_{11}\right|^{2}-\left|S_{22}\right|^{2}}{2\left|S_{12}\right|\left|S_{21}\right|}
$$

and the maximum oscillator power is given by:

$$
P_{o s c}=P_{s a t}\left[1-\frac{1}{G_{o}}-\frac{\operatorname{Ln} G_{O}}{G_{O}}\right]
$$

and the maximum efficient gain $G_{M E}$ is given by:

$$
G_{M E}(\max . \text { oscillator power })=\frac{G_{O}-1}{\operatorname{Ln} G_{O}}
$$

Equations (2) \& ( 5) are used to determine the reduction in $\left|S_{21}\right|$. The modified SParameters are used to design the Oscillator using the linear approach [4] with a few modifications as follows:

(a) A feedback circuit [5] is designed to maximize the input reflection of the oscillator. We use the equation (8) to achieve this condition and calculate the output impedance, which achieves the unconditional stability to the oscillator as shown in Fig.4.

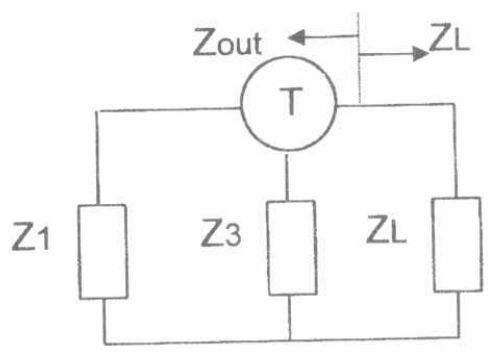

Fig.4 Circuit with series feedback transistor oscillator.

The output impedance is given by:

$$
Z_{\text {out }}=Z_{22}-\frac{Z_{21} Z_{12}}{Z_{11}-Z_{1}}
$$

Where $\mathrm{Zij}_{\mathrm{ij}}$ is the composed of the transistor matrix, and impedance $\mathrm{Z} 3$. The transistor matrix obtained by converting S-Parameters to Z-Parameters [6].

(b) The load circuit design:

$$
\begin{aligned}
& X_{L}=-X_{\text {ouT }} \\
& R_{L}=\frac{-R_{\text {out }}}{3}
\end{aligned}
$$


DESIGN EXAMPLE:

The practical microstrip oscillator circuit is shown in Fig.5. After some calculations we get the initial values of the oscillator parameters as shown in Table 1.

Table 1. the element value of $4.5 \mathrm{GHz}$ oscillator

\begin{tabular}{|c|c|c|c|}
\hline Symbol & Value $[\mathrm{mm}]$ & Symbol & Value $[\mathrm{mm}]$ \\
\hline L1 & 11.766 & L4 & 5.37 \\
L2 & 15.33 & L5 & 11.398 \\
L3 & 15.33 & Zo & $50 \mathrm{ohm}$ \\
\hline
\end{tabular}

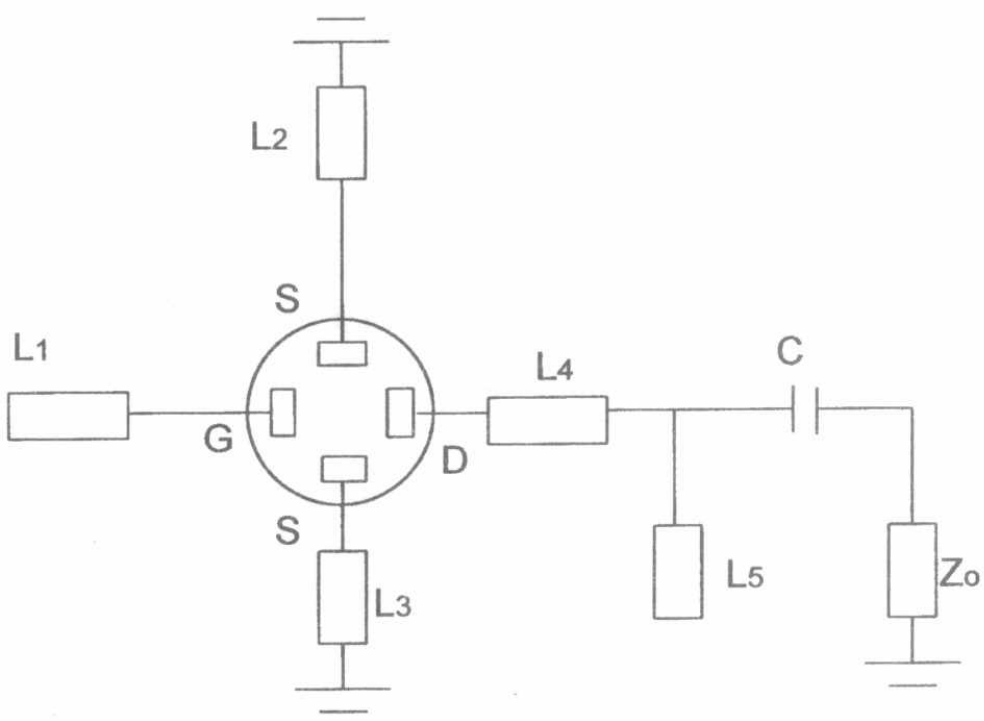

Fig.5 Practical microstrip FET oscillator circuit[7,8]

\section{CAD OF MICROSTRIP OSCILLATORS:}

The Computer Aided Analysis of an oscillator proceeds as follows:

(1) Guessing the oscillation frequency and magnitude. The first guess is entered on the analysis block. Subsequent guesses are derived from the results of previous analysis.

(2) Calculating the voltage drop across the oscillator port as shown in Fig.6.

If the voltage drop is zero, the analysis is finished.

If the phase is zero, the magnitude of the current source is changed.

If the phase is not zero, the frequency of the current source is changed.

(3) Going back to step one.

If there is no voltage drop across the current source, then the current source supplies no power to the circuit. So, the signal must be coming from the oscillator, not the current source, which is our objective. The optimized $4.5 \mathrm{GHz}$ 
oscillator schematic is shown in Fig.7. The results of the loop gain test are presented on two plots as shown in Fig. 8.

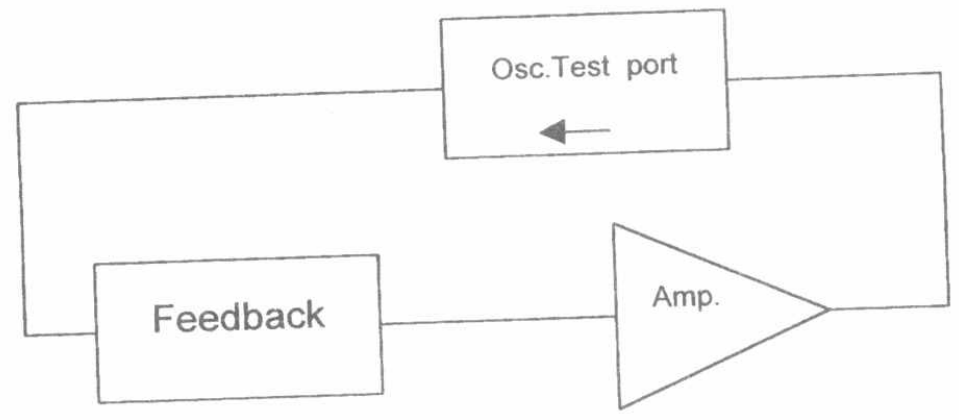

Fig. 6 Oscillator test component in the MDS Program.

(a) A rectangular plot on which two traces are inserted, to show the magnitude and phase of the loop gain S11. If MAG(S11) $>1$ When PHASE (S11) $=0$, The circuit is likely to oscillate at the frequency where the phase is zero. If $\mid$ Loop Gain $\mid<1$, the circuit will not oscillate. Oscillations will build to higher signal levels, where the gain will compress until |Loop Gain| = 1 for large signal operation which is the steadystate case.

(b) A polar plot that shows $\mathrm{S} 11$ in complex form. The trace should encircle the point $(1+j 0)$, with increasing the frequency in clockwise direction. It is clear from Fig. 8 that the circuit will oscillate at about $4.5 \mathrm{GHz}$ frequency.

\section{OSCILLATOR FABRICATION:}

We use GDSII format artwork translator to generate the layout of the oscillator schematic circuit. This layout is shown in Fig.9, with the inclusion of open circuit discontinuity, via effect, metal thickness and device package parasitic, then this layout is translated into Gerber format. The Gerber format is used to generate a transparent mask on a film using a laser photo-plotter with very high accuracy. The microstrip oscillator circuit is built using thin film technology and photolythographic technique and we make all fabrication process on the oscillator circuit, and we chose RT5880 substrates $\left(\varepsilon_{r}=2.2\right)$.

\section{MEASUREMENTS:}

We used in our oscillator measurement a universal test fixture, two power supplie to give us $+V$ for $V_{D S}$ and $-V$ for $V_{G S}$ and spectrum analyzer HP8363A. The output frequency of the oscillator is $4.5305 \mathrm{GHz}$ as shown in Fig.10 and the output spectrum of the $4.5 \mathrm{GHz}$ oscillator is shown in Fig.11, we find, the oscillator is shifted from the ideal one by $+30.5 \mathrm{MHz}$, which represent a good result and the output power is $10 \mathrm{dBm}$. 
Proceedings of the $\mathbf{2}^{\text {nd }}$ ICEENG Conference, 23-25 Nov. 1999

\begin{tabular}{|l|l|}
\hline CS-3 & 8 \\
\hline
\end{tabular}

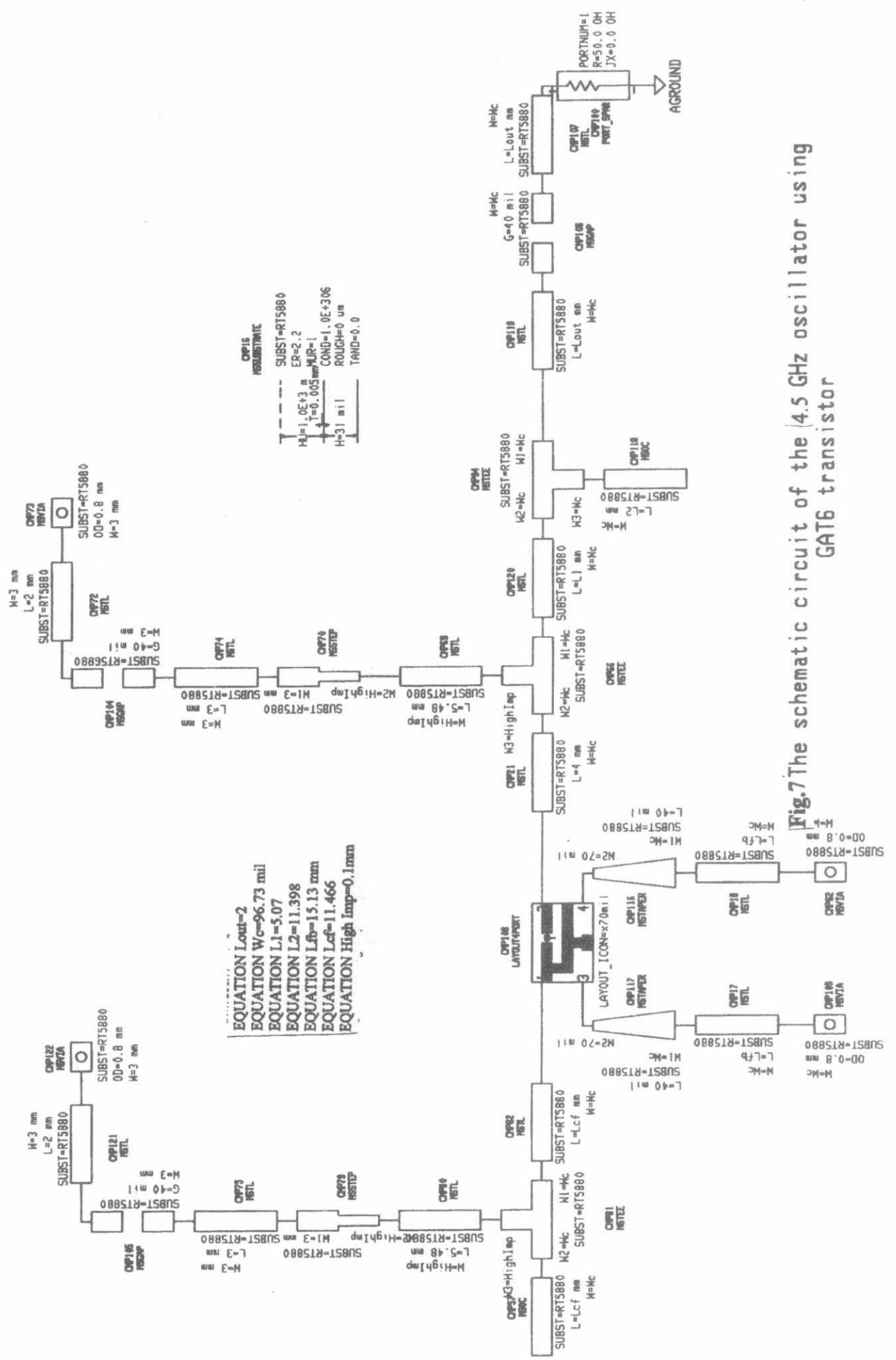


Proceedings of the 2 nd ICEENG Conference, 23-25 Nov. 1999

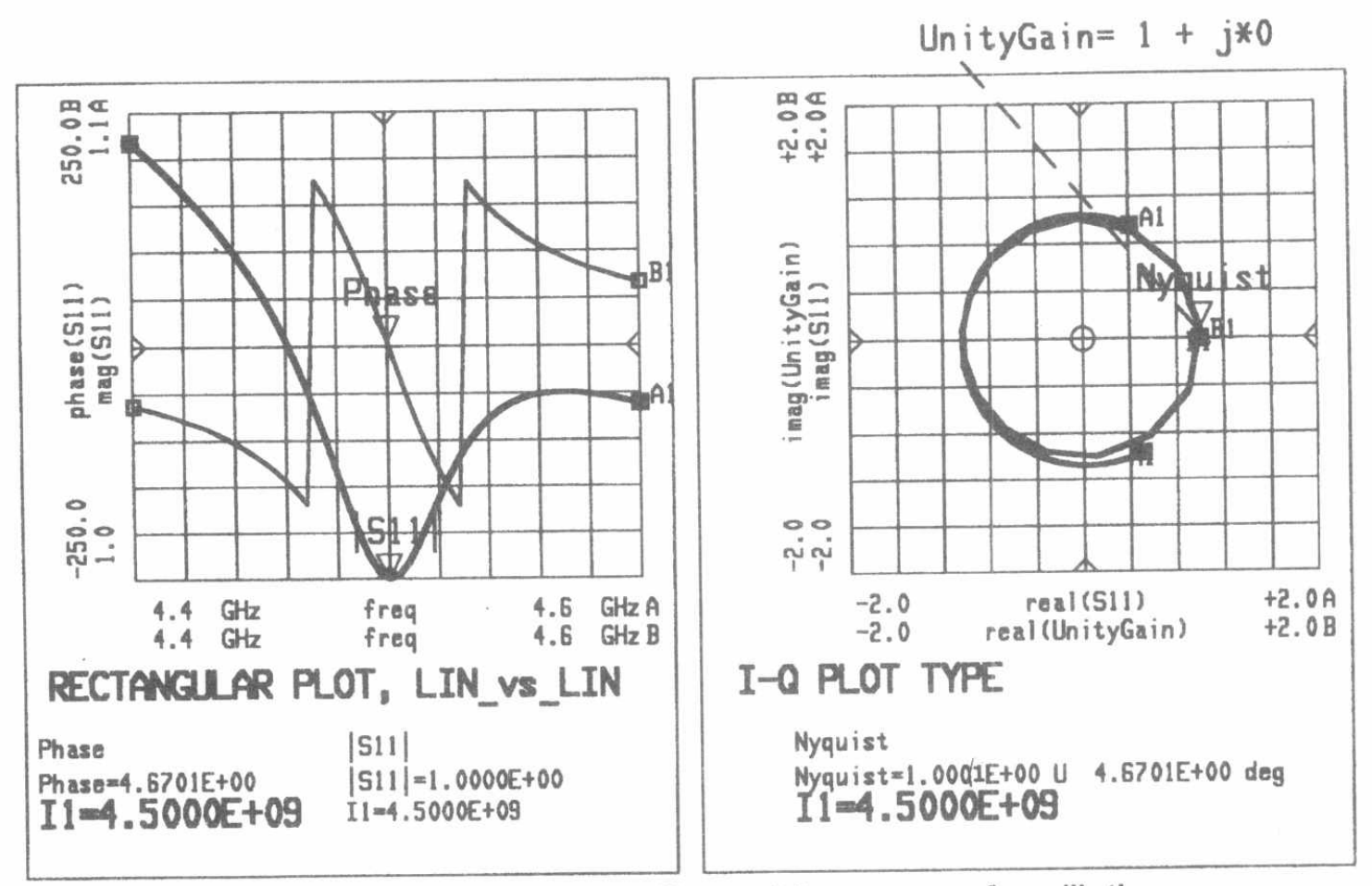

Fig. 8 Estimating the possibility and frequency of oscillation.
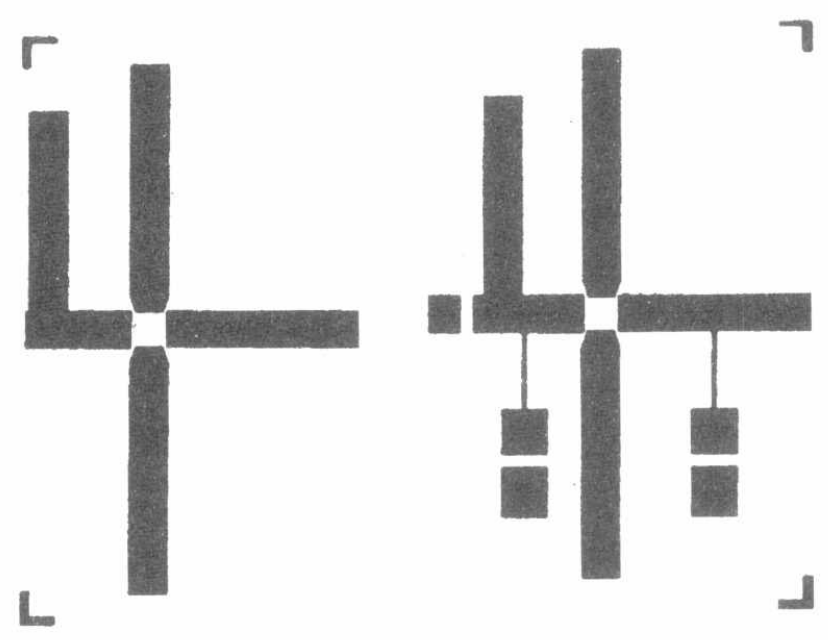

Fig. 9 Layout of the $4.5 \mathrm{GHz}$ oscillator. 
Proceedings of the $\mathbf{2}^{\text {nd }}$ ICEENG Conference, 23-25 Nov. 1999

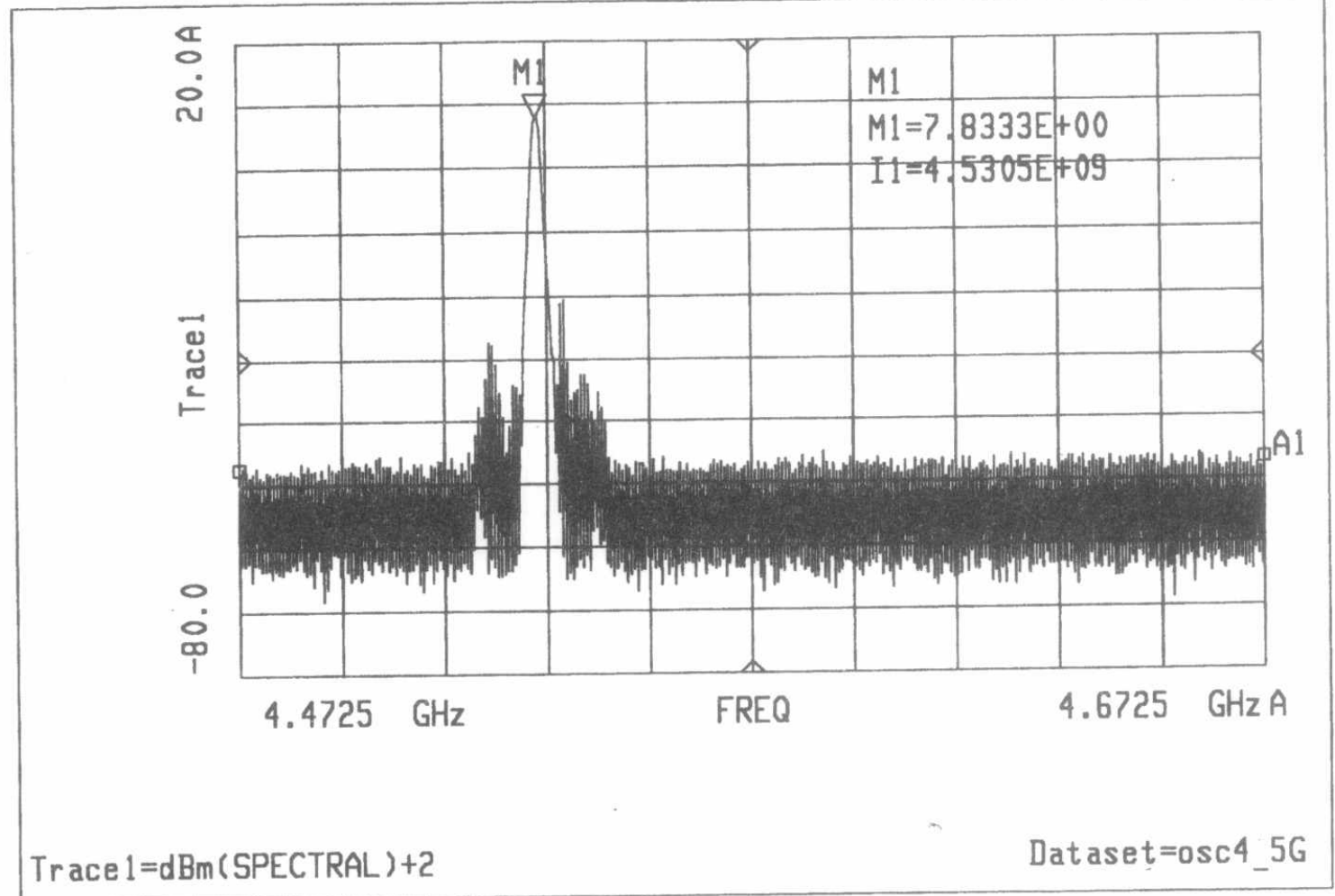

Fig.10 Fundamental frequency of the $4.5 \mathrm{GHz}$ designed oscillator.

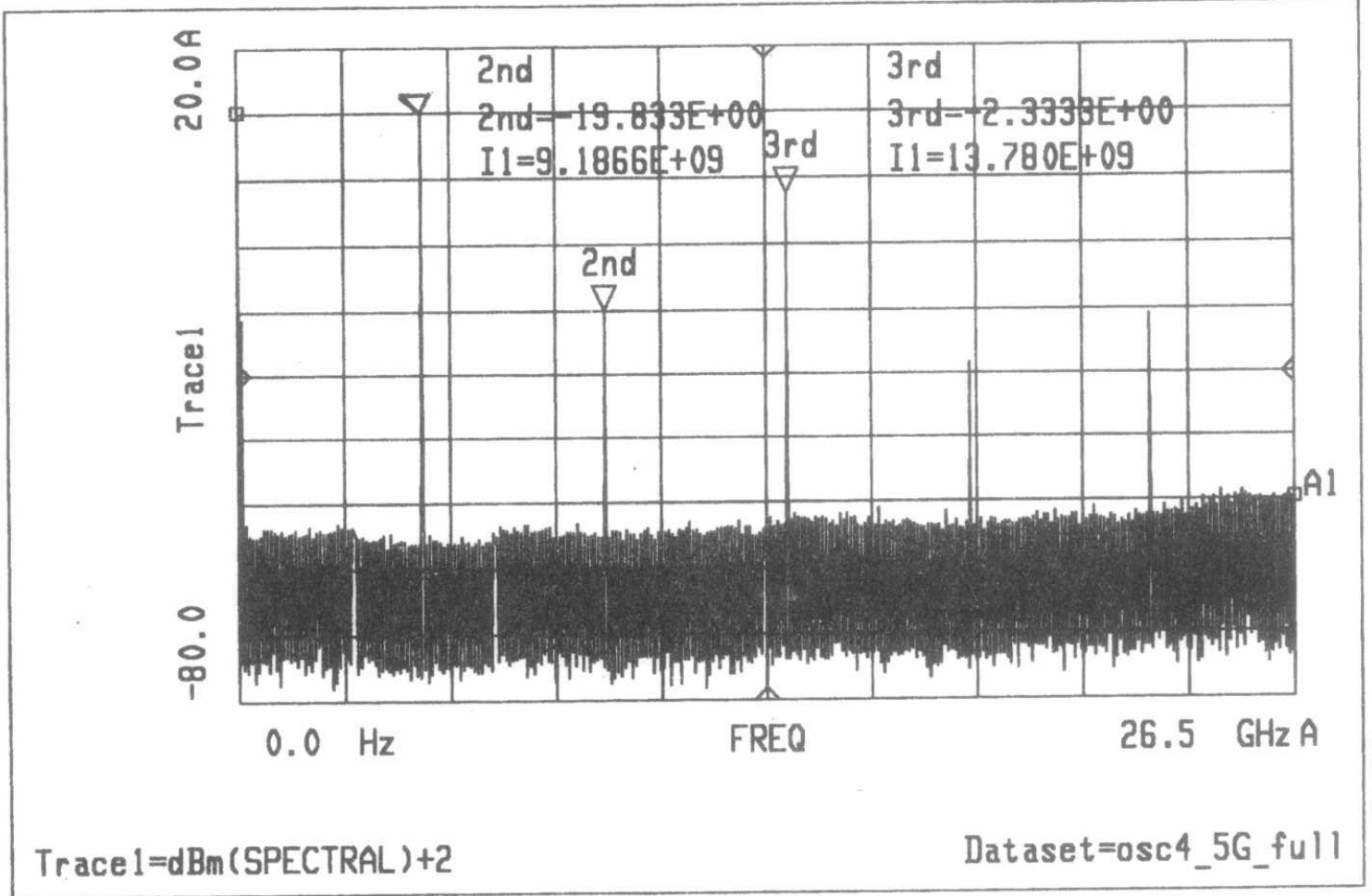

Fig.11 Spectrum analyzer output of the $4.5 \mathrm{GHz}$ oscillator. 
